\title{
Frequency-dependent scaling behavior of polyvinylidene fluoride/metal composites
}

\author{
Maheswar Panda \\ Department of Physics \\ Dr. Harisingh Gour Vishwavidyalaya (A Central University) \\ Sagar 470003, India \\ panda.maheswar@gmail.com
}

Received 6 October 2018; Revised 2 February 2019; Accepted 21 February 2019; Published 8 March 2019

\begin{abstract}
The frequency-dependent percolation and scaling behavior of a variety of polymer/metal composites (PMC), based on polyvinylidene fluoride (PVDF) matrix and various types of fillers such as; metal/alloy particles of different sizes, prepared through cold/hot pressing process conditions have undergone investigation. The universal percolation behavior in the vicinity of percolation threshold $\left(f_{c}\right)$, i.e., $\sigma_{\text {eff }}\left(\omega, f_{\text {con }} \approx f_{c}\right) \propto \omega^{x}$ and $\varepsilon_{\text {eff }}\left(\omega, f_{\text {con }} \approx f_{c}\right) \propto \omega^{-y}$ is well satisfied, which suggests $f_{c}$ to be independent of frequency, where $\sigma_{\text {eff }}$ and $\varepsilon_{\text {eff }}$ are the effective ac conductivity and effective dielectric constants of the composite and $\omega$ is the frequency of applied ac signal. The obtained experimental values of the exponents are consistent with the inter-cluster polarization model $(x=0.72$ and $y=0.28)$, satisfying $x+y=1$. The widely used percolative equations are well fitted with the experimental results of all PMC at all values of the frequency. The value of $f_{c}$ is found to be independent of frequency of the applied signal, suggesting the studied PMC are real percolating systems. The critical exponents $\left(s\right.$ and $\left.s^{\prime}\right)$ which characterize the divergence of $\varepsilon_{\mathrm{eff}}$ and $\sigma_{\mathrm{eff}}$ in the vicinity of $f_{c}$ are found to decrease with the increase of frequency. The rate of decrease of ' $s$ ' and ' $s$ '" with increase of frequency is attributed to the method of preparation, size of the fillers, adhesiveness of polymer/filler and the rate of decrease of $\varepsilon_{\text {eff }}$ with frequency (due to the absence of different extents of contributions of various types of conventional polarizations).
\end{abstract}

Keywords: Composites; critical exponent; frequency; inter-cluster polarization model; percolation; ac conductivity; dielectric constant.

\section{Introduction}

Currently in the twenty first century, novel materials for electrical energy storage applications are undergoing a high demand to be developed. ${ }^{1-5}$ In this regard, the polymer dielectrics (PD) based on ferroelectric polymers such as; polyvinyledene fluoride (PVDF), polyvinyledene fluoride trifluoroethylene will be able to replace the conventional ceramic dielectric materials such as BaTiO3, PMN-PT, etc. ${ }^{6-25}$ In development of these ferroelectric polymer dielectrics (PD), the fillers used are of micron/nano particles of ceramics, metals, carbon nano tubes, carbon black, graphite, graphene, etc. ${ }^{1-30}$ The physics of these PDs falls into two categories. One type of PD is composed of two component polymer/ceramic composites, which are explained by the various effective medium theories. ${ }^{21-23}$ Similarly, the other type of PD is the polymer/metal composites (PMC), where the composites undergo an insulator to metal transition (IMT) at a critical concentration of the conductor called the percolation threshold $\left(f_{c}\right) .{ }^{24-32}$ In particular, as the filler concentration approaches $f_{c}$ from below, the effective a.c. electrical conductivity $\left(\sigma_{\text {eff }}\right)$ and the dielectric constant $\left(\varepsilon_{\text {eff }}\right)$ of the composite increase dramatically which undergoes as a second order phase transition. ${ }^{31}$ The fundamental physics around the IMT of these PD in the vicinity of $f_{c}$ is very interesting both from theoretical and experimental point of view. Percolation theory ${ }^{31}$ states that in the critical phase transition region, the $\varepsilon_{\text {eff }}$ diverges and $\sigma_{\text {eff }}$ increases abnormally at $f_{c}$ and they scales as the following relations:

$$
\begin{gathered}
\varepsilon_{\mathrm{eff}}\left(\omega, f_{\text {con }} \approx f_{c}\right) \propto \omega^{-y}, \\
\sigma_{\mathrm{eff}}\left(\omega, f_{\mathrm{con}} \approx f_{c}\right) \propto \omega^{x}, \\
\varepsilon_{\mathrm{eff}} \propto\left(f_{c}-f_{\mathrm{con}}\right)^{-s} \text { for } f_{\text {con }}<f_{c} \\
\text { or } \quad \varepsilon_{\mathrm{eff}} \propto\left(\frac{\sigma_{\mathrm{con}}}{\sigma_{d}}\right)^{-s} \text { for } f_{\text {con }}<f_{c}, \\
\sigma_{\mathrm{eff}} \propto\left(f_{c}-f_{\text {con }}\right)^{-s^{\prime}} \text { for } f_{\text {con }}<f_{c},
\end{gathered}
$$

where $f_{\text {con }}$ is the volume fraction of conductor in the composite, $\omega$ is the frequency of applied ac signal, $x$ and $y$ are the critical exponents and they are expected to satisfy the relation; $x+y=1 .^{33-36}$ ' $s$ ' is the dielectric exponent in the insulator region. ' $s$ ' is the conductivity exponents in the insulator region. $\sigma_{\mathrm{con}}$ and $\sigma_{d}$ are the conductivity of conductor and insulator, respectively. According to percolation theory, ${ }^{31}$ the values of the critical exponents are only

This is an Open Access article published by World Scientific Publishing Company. It is distributed under the terms of the Creative Commons Attribution 4.0 (CC-BY) License. Further distribution of this work is permitted, provided the original work is properly cited. 
dimensional dependent of the samples and are independent of other factors. The ideal $f_{c}$ value for a three dimensional (3D) ideal system, where the particles are of spherical shape and are of order of micron size is $f_{\text {con }}=0.16 .^{31}$ The 3D universal values of the various exponents are given by; $s_{\text {un }}=0.7-1$ and $s_{\text {un }}^{\prime}=0.7-1 .^{31}$ According to the intercluster polarization model, the $3 \mathrm{D}$ universal values of critical exponents are $x=0.72, y=0.28 .^{33-36}$ Over the last decades of research, due to their achieved better dielectric properties, such as high value of $\varepsilon_{\text {eff }}$ with low value of loss tangent or $\sigma_{\text {eff }}$ and higher dielectric field strength, these PD are going to be the emerging electrical energy storage materials for the current twenty first century for applications in embedded capacitors, printed circuit boards, etc. In a nutshell it can be concluded that the last two decades of research on the PD (two or three component systems comprising of polymer, ceramic, metal, carbon black, carbon fiber, graphite, graphene, carbon nano tube, etc.) has proved them as to be the emerging materials for energy storage, capacitor, low frequency, etc. applications. However, the understanding of the percolation behavior of these PMC remains incomplete and challenging, since the general dependence of percolation and scaling behavior is very rare to find. Generally, it has been seen that the scaling/ percolation behavior of these systems is reported at dc/low frequencies $^{29}$ and interestingly, in some literatures the frequency-dependent percolation and scaling behavior has been reported. ${ }^{30}$ In this paper, I would like to investigate the frequency-dependent percolation and scaling behavior (i.e., how the percolation parameters, such as; percolation threshold, critical exponents and dielectric properties are dependent on the frequency of the applied ac signal) of a general class of PMC based on PVDF matrix and metal/alloy particles of different size, prepared through cold/hot pressing process conditions.

\section{Experimental Details}

The various series of PMC comprising of PVDF and different sizes of $\mathrm{Ni}$ /quasicrystal of $\mathrm{Al}-\mathrm{Cu}-\mathrm{Fe}$ are prepared through different process conditions, e.g., cold press (room temperature consolidation) and hot pressing methods are given in Table 1. The details of the sample preparation and experimental measurements can be found from the earlier published

Table 1. The preparation method and notation for the particular series of PMC under study.

\begin{tabular}{lll}
\hline $\begin{array}{c}\text { Name of the series } \\
\text { of Composites }\end{array}$ & \multicolumn{1}{c}{ Preparation method } & $\begin{array}{c}\text { Notation for the } \\
\text { particular series }\end{array}$ \\
\hline $\mathrm{PVDF} / \mu-\mathrm{Ni}$ & Room temperature consolidation & $\mathrm{A}$ \\
$\mathrm{PVDF} / \mathrm{n}-\mathrm{Ni}$ & Room temperature consolidation & $\mathrm{B}$ \\
$\mathrm{PVDF} / \mu-\mathrm{Ni}$ & Compression hot molding & $\mathrm{C}$ \\
$\mathrm{PVDF} / \mathrm{n}-\mathrm{Ni}$ & Compression hot molding & $\mathrm{D}$ \\
$\mathrm{PVDF} / \mathrm{n}-\mathrm{QC}$ & Room temperature consolidation & $\mathrm{E}$ \\
\hline
\end{tabular}

literatures. $^{12-20}$ All the PMC have undergone electrical and dielectric characterizations.

\section{Results and Discussion}

\subsection{Scaling behavior}

In order to understand the scaling behavior at exactly the $f_{c}$, Eqs. (1) and (2) of percolation theory have been fitted to
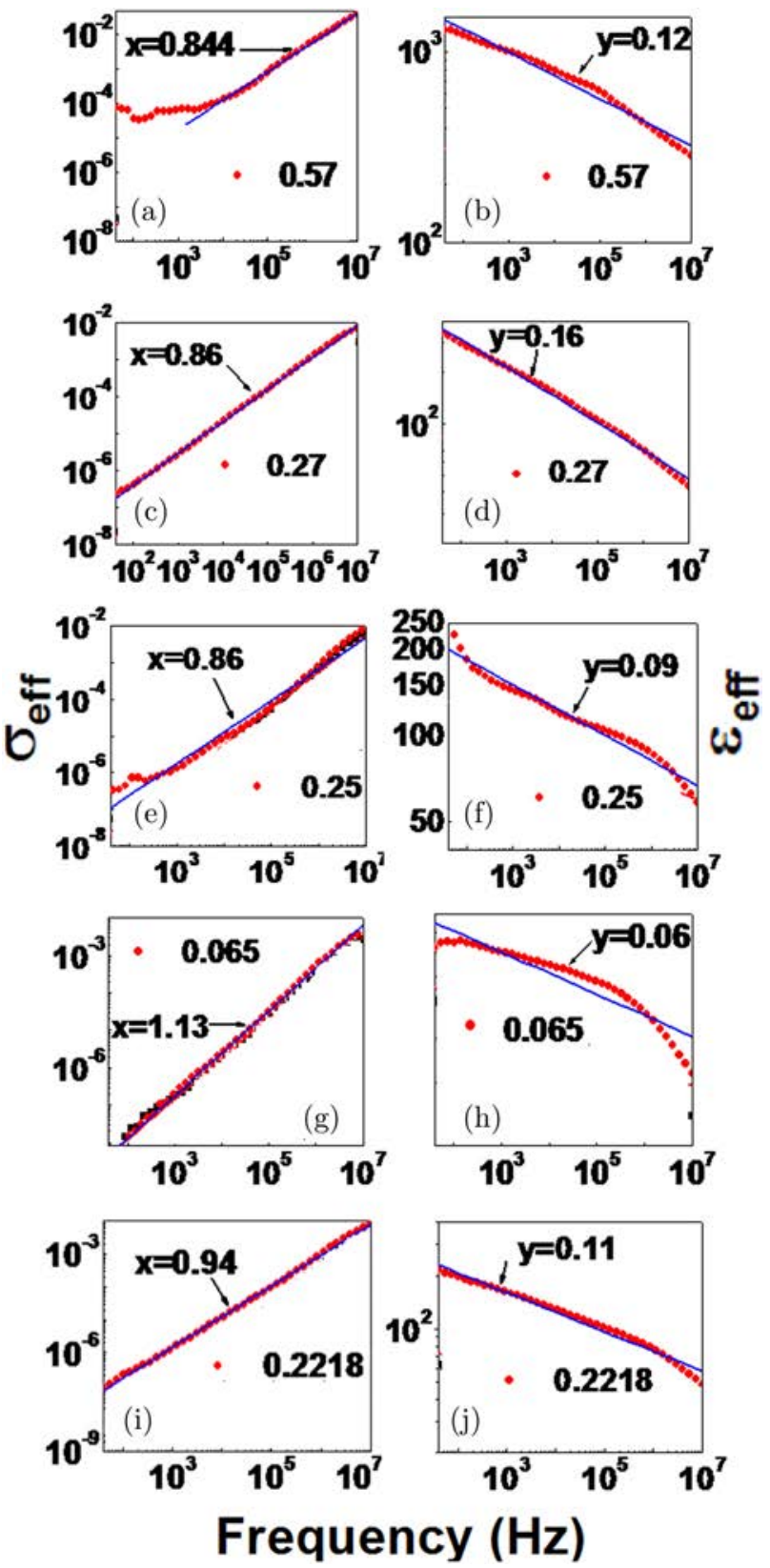

Fig. 1. The fitting of Eqs. (1) and (2) to the experimental results of all the series of PMC to obtain the critical exponents $x$ and $y$ in the vicinity of $f_{c}$, respectively, at $300 \mathrm{~K}$. The solid lines represent the fit to the corresponding experimental data, (a) $x$ of series A, (b) $y$ of series A, (c) $x$ of series B, (d) $y$ of series B, (e) $x$ of series C, (f) $y$ of series C, (g) $x$ of series D, (h) $y$ of series D, (i) $x$ of series E, (j) $y$ of series E. 
Table 2. The values of exponents $x$ and $y$ corresponding to $f_{\text {con }} \approx f_{c}$ for all series of PMC and the verification of intercluster (RC) model, i.e., $x+y=1$.

\begin{tabular}{llllll}
\hline Series & $f_{c}$ & $f_{\text {con }} \approx f_{c}$ & \multicolumn{1}{c}{$x$} & $y$ & $x+y=l$ \\
\hline A & 0.572 & 0.57 & 0.844 & 0.13 & 0.973 \\
B & 0.278 & 0.27 & 0.863 & 0.16 & 1.023 \\
C & 0.259 & 0.25 & 0.863 & 0.09 & 0.953 \\
D & 0.07 & 0.065 & 1.13 & 0.06 & 1.19 \\
E & 0.23 & 0.222 & 0.94 & 0.11 & 1.05 \\
\hline
\end{tabular}

understand the experimental results as a function of the frequencies and are given in Fig. 1 and Table 2, suggesting a very good fit. The critical exponents $x$ and $y$ have been found for the percolative samples and it has been found that the universal scaling behavior of $x+y=1$ at $f_{c}$ best hold good for all the series of PMC. The exponents $(x=0.72$ and $y=0.28$ ) obtained under the inter-cluster polarization model for the case of 3D systems are consistent with the experimental values (Table 1). It can be also observed, that since the Eqs. (1) and (2) are obtained with good fits, for these PMC systems, the value of $f_{c}$ also becomes independent of frequency of the applied ac signal and remains constant as a function of frequency.
Since the exponents ( $s$ and $s^{\prime}$ ) of the scaling laws (3) and (4) are related to $x$ and $y$ under the intercluster polarization (RC) model $^{33-36}$ and in order to see their dependence on the frequency of ac signal, the experimental results of the series A, are fitted to Eqs. (3) and (4) at different frequencies. Equations (3) and (4) shows very good fit with the experimental results at all different frequencies and the obtained values of critical exponents $s$ and $s^{\prime}$ at different frequencies are shown in Fig. 2 and Table 3.

It is observed for series $\mathrm{A}$, the value of $f_{c}$ is found to be 0.57 . The value of the critical exponent ' $s$ ' is found to be 0.72 at $10^{3} \mathrm{~Hz}$ (consistent with its universal value, i.e., $s_{\text {un }}=0.7-1$ ), while the nonuniversal value of ' $s$ ' is also found to be 0.67 and 0.65 for other frequencies such as $10^{5} \mathrm{~Hz}$ and $10^{7} \mathrm{~Hz}$, respectively. Similarly for series $\mathrm{A}$, the value of the critical exponent ' $s$ ' ' is found to be 0.83 at $10^{5} \mathrm{~Hz}$ (consistent with its universal value, i.e., $s_{\text {un }}^{\prime}=0.7-1$ ), while the nonuniversal value of ' $s$ ' ' are found to be 1.15 and 0.64 for other frequencies such as $10^{3} \mathrm{~Hz}$ and $10^{7} \mathrm{~Hz}$ respectively. Thus, I observe, both universal/nonuniversal values of $s$ and $s^{\prime}$ in different frequency domain are obtained for the Series A samples. It is also observed that both the exponents ( $s$ and $\left.s^{\prime}\right)$ are found to frequency dependent [Fig. 2 and Table 3] and their values gradually decreases with increase of frequencies. In order to investigate the general trend of variations in case

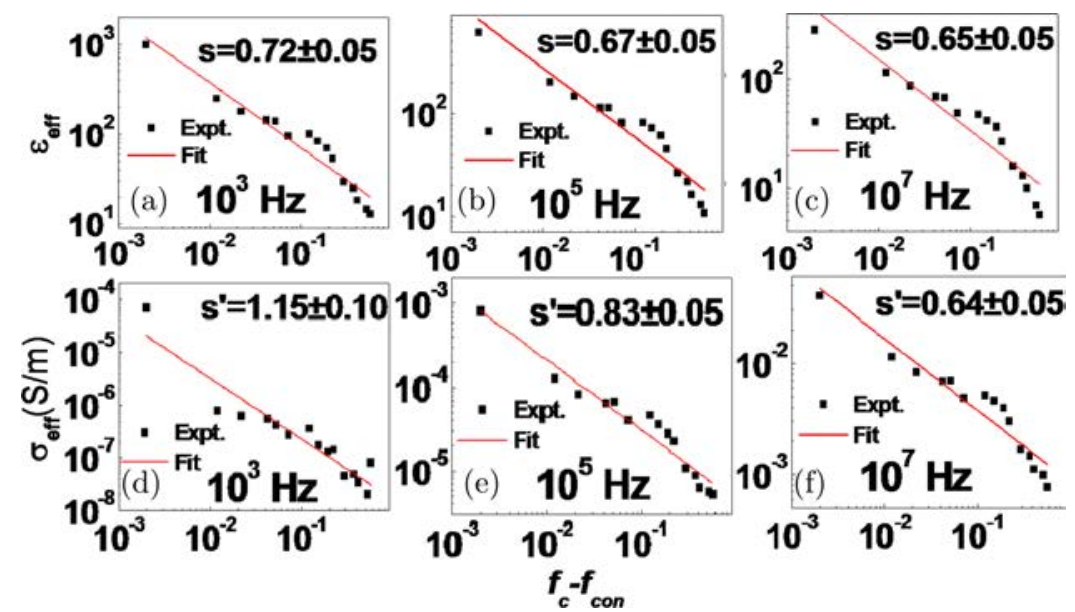

Fig. 2. The fitting of Eqs. (3) and (4) to the experimental results of series A to obtain the critical exponents at $300 \mathrm{~K}$ and at various frequencies, $s$ at (a) $10^{3} \mathrm{~Hz}$, (b) $10^{5} \mathrm{~Hz}$, (c) $10^{7} \mathrm{~Hz}$ and $s^{\prime}$ at, (d) $10^{3} \mathrm{~Hz}$, (e) $10^{5} \mathrm{~Hz}$, (f) $10^{7} \mathrm{~Hz}$.

Table 3. The values of critical exponents $s$ and $s^{\prime}$ for all the series of PMC at different frequencies.

\begin{tabular}{|c|c|c|c|c|c|c|c|c|}
\hline \multirow[b]{2}{*}{ Series } & \multirow[b]{2}{*}{$f_{c}$} & \multirow[b]{2}{*}{$f_{\text {con }} \sim f_{c}$} & \multicolumn{3}{|c|}{$s$} & \multicolumn{3}{|c|}{$s^{\prime}$} \\
\hline & & & $10^{3} \mathrm{~Hz}$ & $10^{5} \mathrm{~Hz}$ & $10^{7} \mathrm{~Hz}$ & $10^{3} \mathrm{~Hz}$ & $10^{5} \mathrm{~Hz}$ & $10^{7} \mathrm{~Hz}$ \\
\hline A & 0.572 & 0.57 & $0.72 \pm 0.05$ & $0.67 \pm 0.05$ & $0.65 \pm 0.05$ & $1.15 \pm 0.10$ & $0.83 \pm 0.05$ & $0.64 \pm 0.05$ \\
\hline B & 0.278 & 0.27 & $0.82 \pm 0.07$ & $0.75 \pm 0.06$ & $0.57 \pm 0.05$ & $1.43 \pm 0.31$ & $1.04 \pm 0.12$ & $0.65 \pm 0.07$ \\
\hline $\mathrm{C}$ & 0.259 & 0.25 & $0.90 \pm 0.10$ & $0.80 \pm 0.13$ & $0.76 \pm 0.14$ & $1.73 \pm 0.20$ & $1.14 \pm 0.16$ & $0.86 \pm 0.13$ \\
\hline D & 0.07 & 0.065 & $0.75 \pm 0.10$ & $0.70 \pm 0.08$ & $0.67 \pm 0.10$ & $1.16 \pm 0.40$ & $0.95 \pm 0.17$ & $0.71 \pm 0.06$ \\
\hline E & 0.23 & 0.23 & $0.69 \pm 0.09$ & $0.61 \pm 0.08$ & $0.59 \pm 0.08$ & $1.09 \pm 0.15$ & $0.80 \pm 0.11$ & $0.65 \pm 0.09$ \\
\hline
\end{tabular}



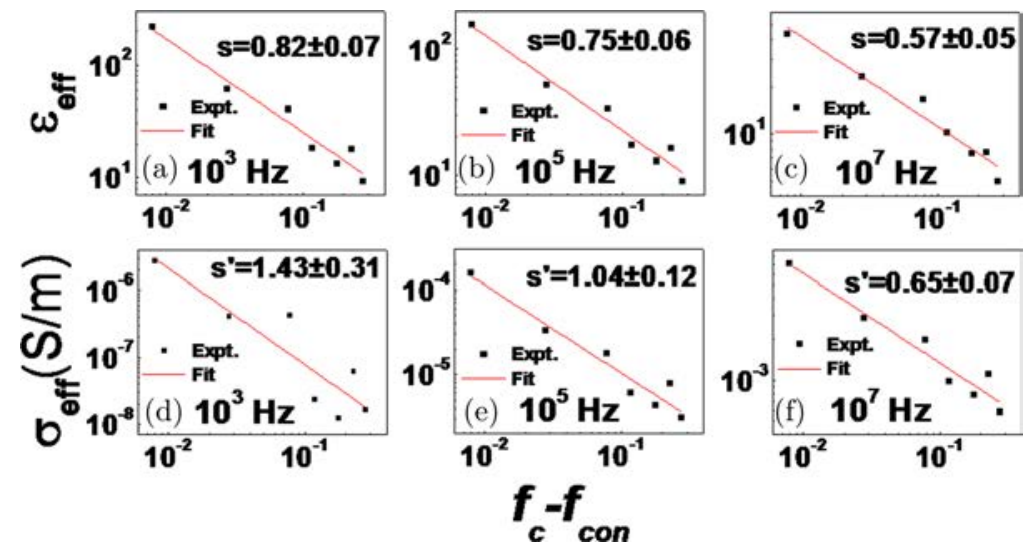

Fig. 3. The fitting of Eqs. (3) and (4) to the experimental results of the series B to obtain the critical exponents at $300 \mathrm{~K}$ and at various frequencies, $s$ at (a) $10^{3} \mathrm{~Hz}$, (b) $10^{5} \mathrm{~Hz}$, (c) $10^{7} \mathrm{~Hz}$ and $s^{\prime}$ at, (d) $10^{3} \mathrm{~Hz}$, (e) $10^{5} \mathrm{~Hz}$, (f) $10^{7} \mathrm{~Hz}$.

of PMC, the same analysis was also extended to all other series of PMC to find a general conclusion and obtained results are given in Table 3 and in the Figs. 3-6 for the series B, C, D and E, respectively.
It is found that, the same type of variation is also observed for all the series of PMC and the equations, Eqs. (3) and (4) shows very good agreement to the experimental results at any selected frequencies. In order to get a clear picture of the
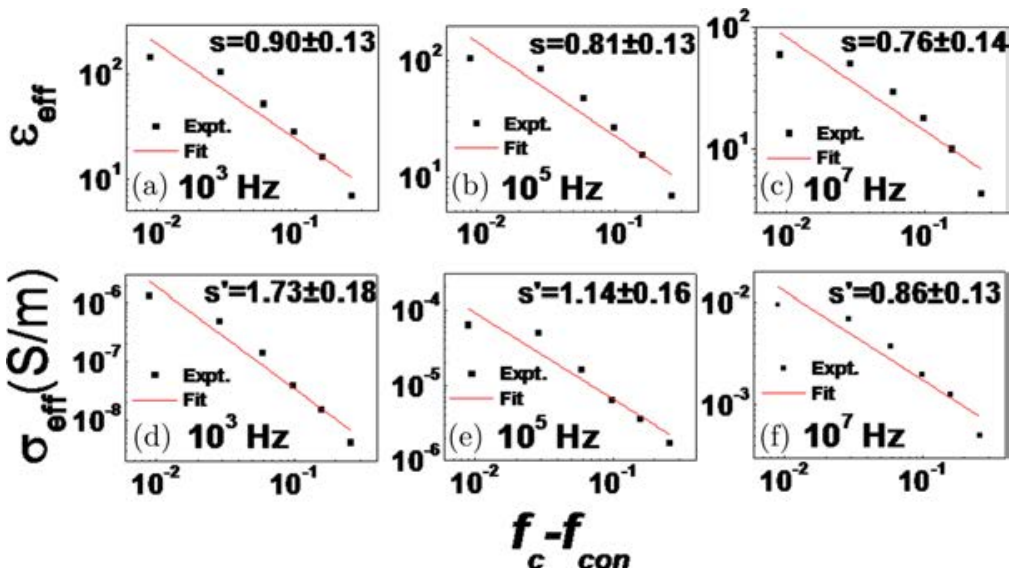

Fig. 4. The fitting of Eqs. (3) and (4) to the experimental results of the series $\mathrm{C}$ to obtain the critical exponents at $300 \mathrm{~K}$ and at various frequencies, $s$ at (a) $10^{3} \mathrm{~Hz}$, (b) $10^{5} \mathrm{~Hz}$, (c) $10^{7} \mathrm{~Hz}$ and $s^{\prime}$ at, (d) $10^{3} \mathrm{~Hz}$, (e) $10^{5} \mathrm{~Hz}$, (f) $10^{7} \mathrm{~Hz}$.
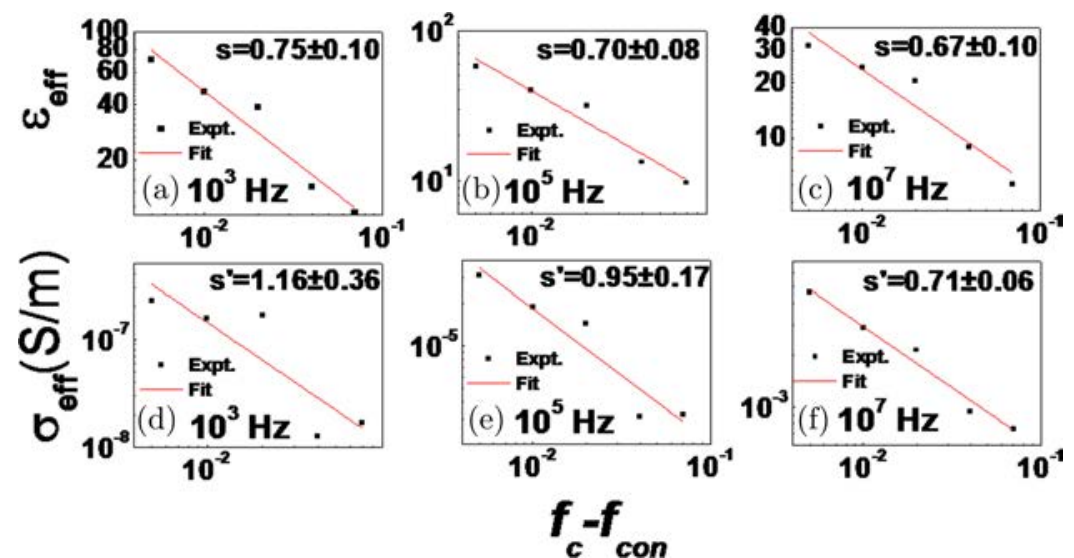

Fig. 5. The fitting of Eqs. (3) and (4) to the experimental results of the series D to obtain the critical exponents at $300 \mathrm{~K}$ and at various frequencies, $s$ at (a) $10^{3} \mathrm{~Hz}$, (b) $10^{5} \mathrm{~Hz}$, (c) $10^{7} \mathrm{~Hz}$ and $s^{\prime}$ at, (d) $10^{3} \mathrm{~Hz}$, (e) $10^{5} \mathrm{~Hz}$, (f) $10^{7} \mathrm{~Hz}$. 

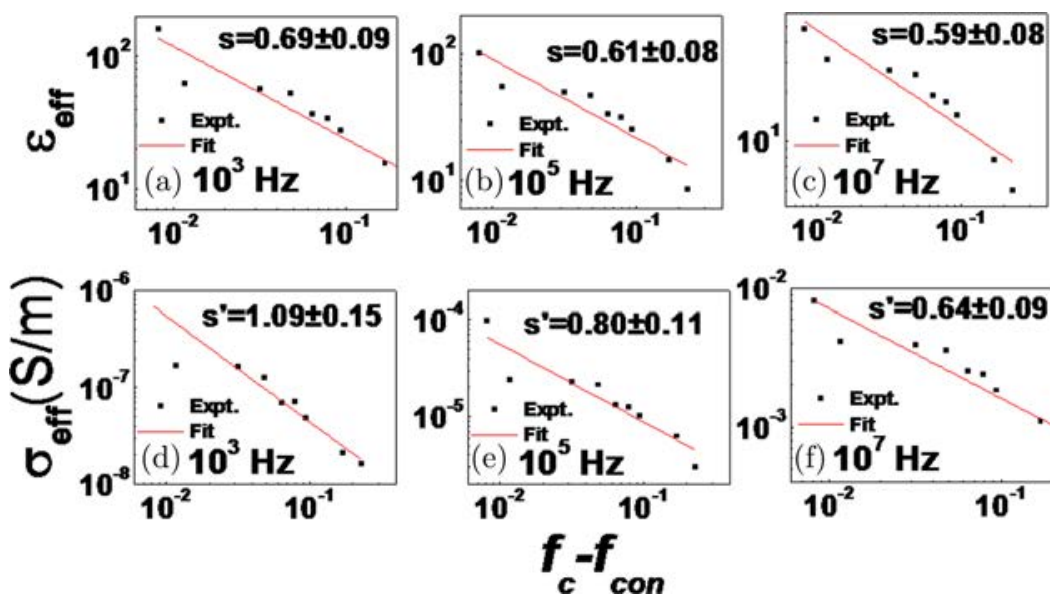

Fig. 6. The fitting of Eqs. (3) and (4) to the experimental results of the series $\mathrm{E}$ to obtain the critical exponents at $300 \mathrm{~K}$ and at various frequencies, $s$ at (a) $10^{3} \mathrm{~Hz}$, (b) $10^{5} \mathrm{~Hz}$, (c) $10^{7} \mathrm{~Hz}$ and $s^{\prime}$ at, (d) $10^{3} \mathrm{~Hz}$, (e) $10^{5} \mathrm{~Hz}$, (f) $10^{7} \mathrm{~Hz}$.

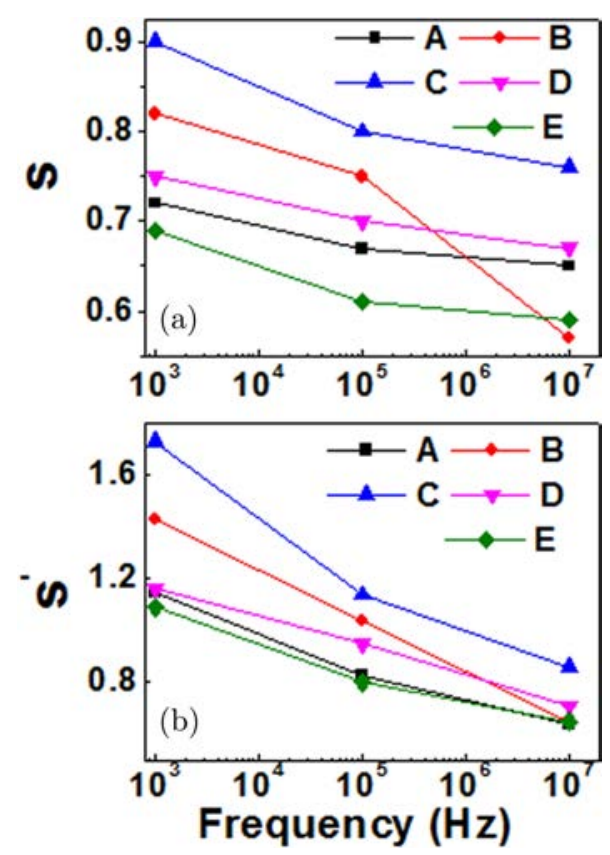

Fig. 7. The variation of critical exponents ' $s$ ' and ' $s$ ', with increase of frequency.

variation of critical exponents $\left(s\right.$ and $\left.s^{\prime}\right)$ as a function of frequency, the data of $s$ and $s^{\prime}$ obtained from the fitting of experimental data of PMC at various frequencies (Figs. 2-6) are given in Fig. 7 for getting a clear picture.

In all PMC, the value of critical exponents $\left(s\right.$ and $\left.s^{\prime}\right)$ are found to be universal as well as non-universal at different frequency domains. The values of $s$ and $s^{\prime}$ are found to decrease with the increase of frequency, while the observation of increase of critical exponents with the increase of frequency have been reported in some other reported dielectric conductor composites. ${ }^{30}$ The decrease of ' $s$ ' and ' $s$ ' with increase of frequency is attributed to the method of preparation, particle size, adhesiveness of polymer/filler and the rate of decrease of $\varepsilon_{\text {eff }}$ with frequency (due to the absence of different extent of contribution of various types polarizations present in the PMC).

\section{Conclusions}

The widely used percolation equations, Eqs. (1)-(4) was used to fit the dielectric and conductivity data of 5 PMC systems. The different PMC gave different values of $f_{c}$ with different extent of enhancement of $\sigma_{\text {eff }}$ and $\varepsilon_{\text {eff }}$ at their respective $f_{c}$ for different frequencies. The values of $f_{c}$ are found to be nonuniversal as compared to the theoretically predicted ideal value. ${ }^{31}$ The nonuniversal values of $f_{c}$ for different PMC has been attributed to a variety of parameters, such as; size, shape, process conditions, etc. The value of $f_{c}$ is found to be independent of frequency of the applied ac signal, since the universal percolation equations are satisfied in the vicinity of IMT with $x+y=1$, suggests the investigated PMC systems are real percolating systems. The analysis of the experimental data of a variety of different class of PMC, confirms the obtained scaling exponents $\left[s\right.$ and $\left.s^{\prime}\right]$ to be both universal and nonuniversal and are dependent on the frequency of the applied ac signal. The decrease of critical exponents ' $s$ ' and ' $s$ ', with increase of frequency is attributed to the method of preparation, size of fillers, adhesiveness of polymer/filler and the rate of decrease of $\varepsilon_{\text {eff }}$ with frequency in case of all these reported PMC.

\section{Acknowledgments}

The financial support received from the UGC-BSR project [Grant No: F.30-12/2014(BSR)] and DST (SERB) project [Grant No: SR/FTP/PS-177/2011] are gratefully acknowledged. 


\section{References}

${ }^{1}$ V. K. Thakur and M. R. Kessler, Polymer Nanocomposites: New Advanced Dielectric Materials for Energy Storage Applications, in Advanced Energy Materials (John Wiley \& Sons, Inc., Hoboken, NJ, USA, 2014).

${ }^{2}$ Q. Li, L. Chen, M. R. Gadinski, S. Zhang, G. Zhang, H. U. Li, E. Iagodkine, A. Haque, L. Q. Chen, T. N. Jackson and Q. Wang, Flexible high-temperature dielectric materials from polymer nanocomposites, Nature 523, 576 (2015).

${ }^{3}$ V. Sharma, C. Wang, R. G. Lorenzini, R. Ma, Q. Zhu, D. W. Sinkovits, G. Pilania, A. R. Oganov, S. Kumar, G. A. Sotzing, S. A. Boggs and R. Ramprasad, Rational design of all organic polymer dielectrics, Nature Comm. 5, 48451 (2014).

${ }^{4}$ Prateek, V. K. Thakur and R. K. Gupta, Recent progress on ferroelectric polymer-based nanocomposites for high energy density capacitors: Synthesis, dielectric properties, and future aspects, Chem. Rev. 116, 4260 (2016).

${ }^{5}$ Q. Li, F. Z. Yao, Y. Liu, G. Zhang, H. Wang and Q. Wang, HighTemperature dielectric materials for electrical energy storage, Annu. Rev. Mater. Res. 48, 219 (2018).

${ }^{6}$ M. Yang, H. Zhao, D. He and J. Bai, Largely enhanced dielectric properties of carbon nanotubes/polyvinylidene fluoride binary nanocomposites by loading a few boron nitride nanosheets, Appl. Phys. Lett. 109, 072906 (2016).

${ }^{7}$ Z. M. Dang, J. K. Yuan, S. H. Yao and R. J. Liao, Flexible nanodielectric materials with high permittivity for power energy storage, Adv. Mater. 25, 6334 (2013).

${ }^{8}$ Z. M. Dang, L. Wang, Y. Yin, Q. Zhang and Q. Q. Lei, Giant dielectric permittivities in functionalized carbon-nanotube/ electroactive-polymer nanocomposites, Adv. Mater. 19, 852 (2007).

${ }^{9}$ X. Shan, L. Zhang, X. Yang and Z. Y. Cheng, Dielectric composites with a high and temperature-independent dielectric constant, J. Adv. Ceramics 1, 310 (2012).

${ }^{10}$ L. Zhang, X. Shan, P. Bass, Y. Tong, T. D. Rolin, C. W. Hill, J. C. Brewer, D. S. Tucker and Z.-Y. Cheng, Process and microstructure to achieve ultra-high dielectric constant in ceramic-polymer composites, Sci. Rep. 6, 35763 (2016).

${ }^{11}$ L. Zhang, Z. Liu, X. Lu, G. Yang, X. Zhang and Z. Y. Cheng, Nano-clip based composites with a low percolation threshold and high dielectric constant, Nano Energy 26, 550 (2016).

${ }^{12} \mathrm{M}$. Panda, Major role of process conditions in tuning the percolation behavior of polyvinylidene fluoride based polymer/metal composites, Appl. Phys. Lett. 111, 0429051 (2017).

${ }^{13}$ M. Panda, V. Srinivas and A. K. Thakur, Role of polymer matrix in large enhancement of dielectric constant in polymer-metal composites, Appl. Phys. Lett. 99, 0429051 (2011).

${ }^{14}$ M. Panda, V. Srinivas and A. K. Thakur, Dielectric spectroscopy of polymer-metal composites across the percolation threshold, J. Adv. Dielectr. 4, 1450027 (2014).

${ }^{15}$ M. Panda, V. Srinivas and A. K. Thakur, Universal microstructure and conductivity relaxation behaviour of polymer-conductor composites across the percolation threshold, Current Appl. Phys. 14, 1596 (2014).

${ }^{16} \mathrm{M}$. Panda, V. Srinivas and A. K. Thakur, Surface and interfacial effect of filler particle on electrical properties of polyvinyledene fluoride/nickel composites, Appl. Phys. Lett. 93, 2429081 (2008).

${ }^{17}$ M. Panda, V. Srinivas and A. K. Thakur, On the question of percolation threshold in polyvinylidene fluoride/nanocrystalline nickel composites, Appl. Phys. Lett. 92, 1329051 (2008).
${ }^{18}$ M. Panda, A. Mishra and P. Shukla, Effective enhancement of dielectric properties in cold-pressed polyvinyledene fluoride/barium titanate nanocomposites, SN Applied Sciences 1, 230 (2019).

${ }^{19}$ M. Panda, V. Srinivas and A. K. Thakur, Percolation behavior of polymer/metal composites on modification of filler, Mod. Phys. Lett. B 28, 1450055 (2014).

${ }^{20} \mathrm{M}$. Panda, Evidence of a third kind of Johnscher's like universal dielectric response, J. Adv. Dielect. 8, 1850028 (2018).

${ }^{21}$ Y. Bai, Z. Y. Cheng, V. Bharati, H. S. Xu and Q. M. Zhang, Highdielectric-constant ceramic-powder polymer composites, Appl. Phys. Lett. 76, 3804 (2000).

${ }^{22}$ T. Yamada, T. Ueda and T. Kitayama, Piezoelectricity of a highcontent lead zirconate titanate/polymer composite, J. Appl. Phys. 53, 4328 (1982).

${ }^{23}$ J. W. Zha, Z. M. Dang, T. Yang, T. Zhou, H. T. Song and S. T. Li, Advanced dielectric properties of $\mathrm{BaTiO}_{3} /$ polyvinylidene-fluoride nanocomposites with sandwich multi-layer structure, IEEE Trans. Dielectr. Electr. Insul. 19, 1312 (2012).

${ }^{24}$ L. Zhang, X. Wang and Z. Y. Cheng, A case study of conductordielectric 0-3 composites using Ni-P (VDF-CTFE) nanocomposites, J. Adv. Phys. 4, 362 (2015).

${ }^{25}$ L. Zhang and Z. Y. Cheng, Development of polymer-based 0-3 composites with high dielectric constant, J. Adv. Dielectrics. 1, 389 (2011).

${ }^{26}$ L. Zhang, W. Wang, X. Wang, P. Bass and Z. Y. Cheng, Metalpolymer nanocomposites with high percolation threshold and high dielectric constant, Appl. Phys. Lett. 103, 232903 (2013).

${ }^{27}$ L. Zhang, P. Bass and Z. Y. Cheng, Revisiting the percolation phenomena in dielectric composites with conducting fillers, Appl. Phys. Lett. 105, 042905 (2014).

${ }^{28}$ L. Zhang, P. Bass, G. Wang, Y. Tong, Z. Xu and Z. Y. Cheng, Dielectric response and percolation behavior of $\mathrm{Ni}-\mathrm{P}$ (VDFTrFE) nanocomposites, J. Adv. Dielectr. 7, 1750015 (2017).

${ }^{29}$ M. Panda, V. Srinivas and A. K. Thakur, Non-universal scaling behavior of polymer-metal composites across the percolation threshold, Res. in Phys. 5, 136 (2015).

${ }^{30}$ L. Zhang, P. Bass, Z. M. Dang and Z. Y. Cheng, Characterization of percolation behavior in conductor-dielectric 0-3 composites, J. Adv. Dielectr. 4, 1450035 (2014).

${ }^{31}$ D. Stauffer and A. Aharony, Introduction to Percolation Theory (Tayler and Francis, London, 1992).

${ }^{32}$ M. Panda, Scaling and Relaxation behavior of Polymer-metal composites across the Percolation Threshold, Ph.D. Thesis, IIT, Kharagpur (2010).

${ }^{33}$ D. Wilkinson, J. S. Langer and P. N. Sen, Enhancement of the dielectric constant near a percolation threshold, Phys. Rev. B 28, 1081 (1983).

${ }^{34}$ Y. Song, T. W. Noh, S. I. Lee and J. R. Gaines, Experimental study of the three dimensional ac conductivity and dielectric constant of conductor-insulator composite near the percolation threshold, Phys. Rev. B 33, 904 (1986).

${ }^{35}$ D. J. Bergman and Y. Imry, Critical behavior of the complex dielectric constant near the percolation threshold of a heteogenous material, Phys. Rev. Lett. 39, 1222 (1977).

${ }^{36}$ D. M. Grannan, J. C. Garland and D. B. Tanner, Critical behavior of the dielectric constant of a random composite near the percolation threshold, Phys. Rev. Lett. 46, 375 (1981). 